\title{
Pocket-Sized Ultrasound for Physical Diagnosis
}

\author{
Jason C. Ojeda, MD FACP' , James Colbert, $M D^{2}$, and Maria Yialamas, MD ${ }^{3}$ \\ 'Department of Internal Medicine, Thomas Jefferson University, Philadelphia, PA, USA; ${ }^{2}$ Department of Medicine, Newton Wellesley Hospital, \\ Newton, MA, USA; ${ }^{3}$ Department of Medicine, Brigham and Women's Hospital, Boston, MA, USA.
}

$\mathrm{J}$ Gen Intern Med 30(7):884

DOI: $10.1007 / \mathrm{s} 11606-015-3319-1$

(c) Society of General Internal Medicine 2015

The authors reply-

We appreciate Drs. Galen and Schnobrich's comments on our work. While we acknowledge that studies have shown that internal medicine residents are able to develop the skills necessary to acquire and interpret ultrasound images given sufficient time in training, ${ }^{1,2}$ it was our desire to design an intervention that could be widely implemented with minimal investment of time and resources. Emergency medicine has well-established ultrasound fellowships, and clinical faculty are in a position to supervise and assist in the development of residents' skills. Outside of critical care and cardiology, internal medicine largely lacks faculty trained in the use of ultrasound, and thus residents have few opportunities for supervised practice of ultrasound skills. Given that ultrasound use is not currently widespread among internal medicine attending physicians, we felt that studying whether a time-efficient program would provide residents with sufficient training was particularly relevant. The curriculum we tested included a short didactic session followed by independent practice, and could be promptly implemented by internal medicine residency programs without significant redesign of the overall training program. Thus we limited our training to 3 hours, which others have shown to be sufficient for training, albeit for a more limited set of ultrasound skills. ${ }^{3,4}$

Our trial focused on the evaluation of ambulatory patients rather than critical care patients, where the utility of ultrasound has already been well documented in the literature. ${ }^{5}$ We agree that some of the clinical findings in our study, such as the assessment of hepatosplenomegaly, are advanced skills, but we chose to include findings that are routinely assessed during a standard physical exam, as such findings are relevant when assessing the incremental benefit of ultrasound over physical diagnosis. Detecting differences in individual exam abnormalities was not a pre-specified outcome of our study, yet when this was performed, significant differences between the ultrasound and physical exam groups were found only for aortic regurgitation and for left ventricular enlargement. These differences were no longer significant when adjusted for multiple testing.

We agree with Drs. Galen and Schnobrich that more studies will be needed to determine how best to incorporate point-ofcare ultrasound into residency training and which physical exam findings should be emphasized. Our study found that a brief point-of-care ultrasound training session was not sufficient for internal medicine residents to augment their overall diagnostic skills. These results suggest that developing ultrasound competence among internal medicine residents will likely require a substantial investment of both time and resources.

Conflict of Interest: The authors declare that they do not have a conflict of interest.

Corresponding Author: Maria Yialamas, MD; Department of MedicineBrigham and Women's Hospital, 70 Francis Street, Boston, MA 02115, USA (e-mail: MYIALAMAS@PARTNERS.ORG).

\section{REFERENCES}

1. Schnobrich DJ, Olson APJ, Broccard A, Duran-Nelson A. Feasibility and acceptability of a structured curriculum in teaching procedural and basic diagnostic ultrasound skills to internal medicine residents. J Grad Med Educ. 2013;5:493-7.

2. Chalumeau-Lemoine $\mathbf{L}$, et al. Results of short-term training of naïve physicians in focused general ultrasonography in an intensive-care unit. Intensive Care Med. 2009;35:1767-1771.

3. Alexander $\mathbf{J H}$, et al. Feasibility of point-of-care echocardiography by internal medicine house staff. Am Heart J. 2004; 147:476-481.

4. Panoulas VF, et al. Pocket-size hand-held cardiac ultrasound as an adjunct to clinical examination in the hands of medical students and junior doctors. Eur Heart J - Cardiovasc Imaging. 2013;14:323-330.

5. Vignon $\mathbf{P}$, et al. Focused training for goal-oriented hand-held echocardiography performed by noncardiologist residents in the intensive care unit. Intensive Care Med. 2007;33:1795-1799. 